\title{
Articular cartilage regeneration with autologous peripheral blood stem cells versus hyaluronic acid: a randomized controlled trial
}

\begin{abstract}
PURPOSE:The purpose of this study was to compare histologic and magnetic resonance imaging (MRI) evaluation of articular cartilage regeneration in patients with chondral lesions treated by arthroscopic subchondral drilling followed by postoperative intra-articular injections of hyaluronic acid (HA) with and without peripheral blood stem cells (PBSC). METHODS:Fifty patients aged 18 to 50 years with International Cartilage Repair Society (ICRS) grade 3 and 4 lesions of the knee joint underwent arthroscopic subchondral drilling; 25 patients each were randomized to the control (HA) and the intervention (PBSC $+\mathrm{HA}$ ) groups. Both groups received 5 weekly injections commencing 1 week after surgery. Three additional injections of either HA or PBSC + HA were given at weekly intervals 6 months after surgery. Subjective IKDC scores and MRI scans were obtained preoperatively and postoperatively at serial visits. We performed second-look arthroscopy and biopsy at 18 months on 16 patients in each group. We graded biopsy specimens using 14 components of the International Cartilage Repair Society Visual Assessment Scale II (ICRS II) and a total score was obtained. MRI scans at 18 months were assessed with a morphologic scoring system. RESULTS:The total ICRS II histologic scores for the control group averaged 957 and they averaged 1,066 for the intervention group $(\mathrm{P}=.022)$. On evaluation of the MRI morphologic scores, the control group averaged 8.5 and the intervention group averaged 9.9 $(\mathrm{P}=.013)$. The mean 24-month IKDC scores for the control and intervention groups were 71.1 and 74.8 , respectively $(\mathrm{P}=.844)$. One patient was lost to follow-up. There were no notable adverse events. CONCLUSIONS:After arthroscopic subchondral drilling into grade 3 and 4 chondral lesions, postoperative intra-articular injections of autologous PBSC in combination with HA resulted in an improvement of the quality of articular cartilage repair over the same treatment without PBSC, as shown by histologic and MRI evaluation. LEVEL OF EVIDENCE:Level II, randomized controlled trial (RCT).
\end{abstract}

Keyword: Articular cartilage regeneration; Autologous peripheral blood stem cells; Hyaluronic acid; Blood stem cells 